Historic, Archive Document

Do not assume content reflects current scientific knowledge, policies, or practices. 



\section{SPRING 1917}

WHOLESALE CATALOGUE

\section{LEESLEY BROTHERS} .

\section{N UR S E R I E S}

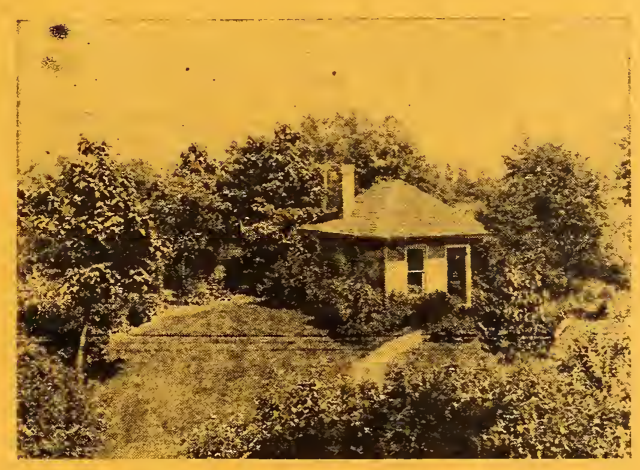

NURSERY AND OFFICE

Crawford and Peterson Avenues

Phone Monticello 48

CHICAGO, ILLINOIS 
Hort. and Pom. No. 201.

\section{U. S. DEPARTMENT OF AGRICULTURE.}

Official Business,

Penalty for Private Use, $\$ 300$.

U. S. Department of Agriculture,

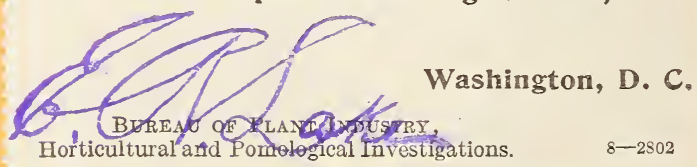




\section{WHOLESALE CATALOGUE SPRING 1917}

\section{LEESLEY BROTHERS}

NURSERIES

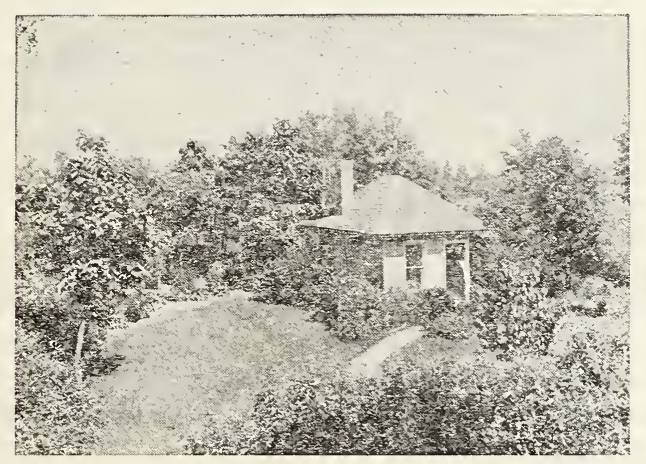

NURSERY AND OFFICE

Crawford and Peterson Avenues

Phone Monticello 48

CHICAGO, ILLINOIS 


\section{LEESLEY BROTHERS N U R S E R IE S}

Are situated in the northern part of the City Limits, in one of the most fertile districts in Illinois. Our Nurseries are easily reached either by the Northwestern Railroad, with stations on both sides of the Nurseries, or the North Crawford Ave. Car Line, which runs within one-half mile of our grounds.

Two branches of the Northwestern Railroad running through our grounds with a private switch, warehouse and packing sheds, the Chicago, Milwaukee and St. Paul R. R. at a 'short distance, all of the Express Companies near at hand offer us unsurpassed facilities for shipping stock. In addition to this we have installed two motor trucks to insure quick deliveries. Where the distance from the nursery is very great we shall make a small charge for cartage, but this will always be specified before delivery is made.

\section{R E M I T T A N C E S}

should be made by Postal or Express Money Orders, Bank Drafts on Chicago or by Registered Letters made payable to the order of LEESLEY BROS.

\section{$\begin{array}{llllll}P & R & I & C & E & S\end{array}$}

do not include packing, which will be charged for at cost. Five will be furnished at the 10 rate and 25 at the 100 rate. No charge for delivery to the express companies or railroads. Certificate of inspection furnished with each shipment. 


\section{ORNAMENTAL TREES}

ACER dasycarpum (Silver-leaved Maple)

8 to 10 -feet................. \$0.35

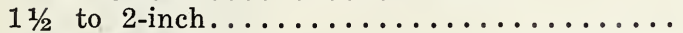

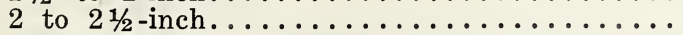

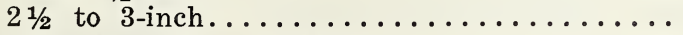

platanoides (Norway Maple)

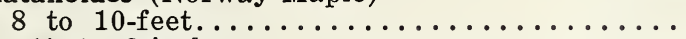

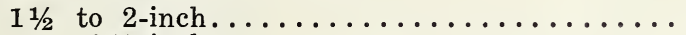

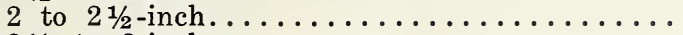

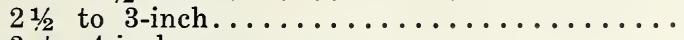

3 to 4 -inch.

saccharum (Sugar or Rock Maple)

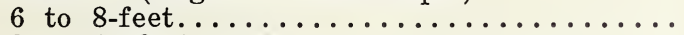

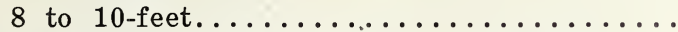

Schwedlerii (Purple-leaved Norway Maple)

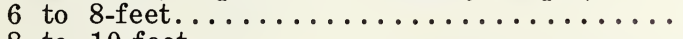

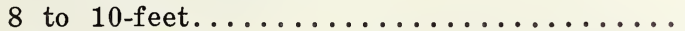

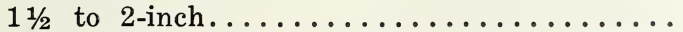

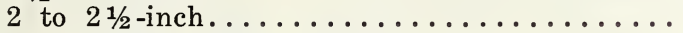

Wierii (Wier's Cut-leaved Maple)

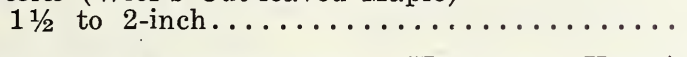

AESCULUS Hippocastanum (European Horse) Chestnut)

$1 \frac{1}{2}$ to 2 -inch.

AILANTHUS glandulosa (Tree of Heaven)

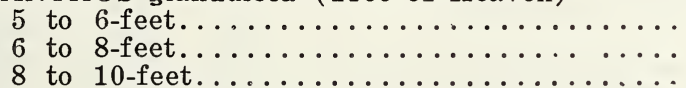

BETULA papyracea (Paper or Canoe Birch)

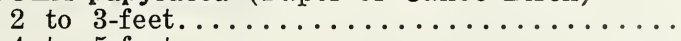

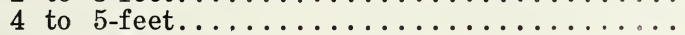

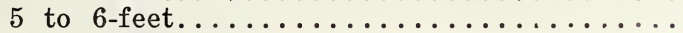

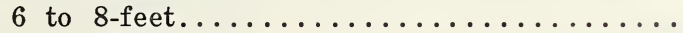

8 ot 10 -feet.

CATALPA Bungei (Chinese)

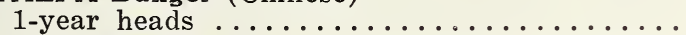

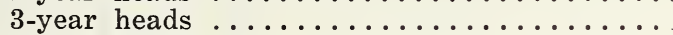

4-year heads

speciosa (Western Catalpa)

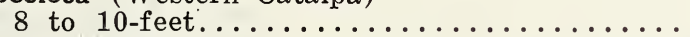

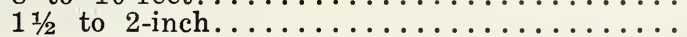

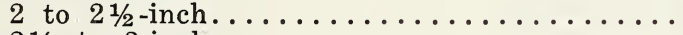

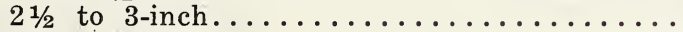

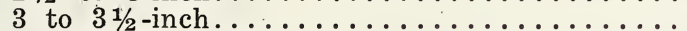

5 -inch

6-inch

8-inch

CELTIS occidentalis (Hackberry)

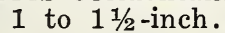

1.00

CERCIS Canadensis (American Judas Tree)

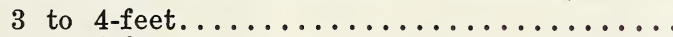

5 to 6 -feet.

FRAXINUS Americana (American White Ash)

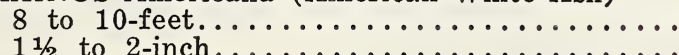

$21 / 2$-inch

.50

1.00

1.25

3 -inch
7.50

17.50 25.00

4.00

Per 10 Per 100

$\$ 25.00$

40.00

75.00

40.00

85.00

135.00

200.00

40.00

50.00

60.00

100.00

135.00

85.00

85.00

15.00

30.00

12.00

25.00

30.00

40.00

60.00

60.00

85.00

100.00

25.00

45.00

75.00

90.00

10.00

8.50

30.00

10.00

17.50

75.00 


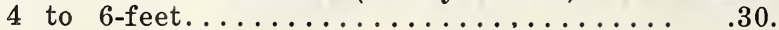

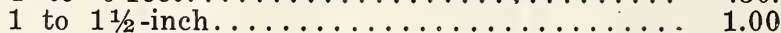

7.50

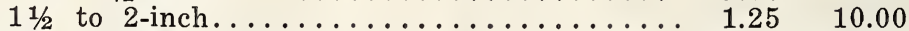

50.00

2 to $2 \frac{1}{2}$-inch

2.00

15.00

$21 / 2$ to 3 -inch.

3.00

GYMNOCLADUS Canadensis (Kentucky Coffee Tree)

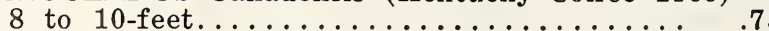

25.00

JUGLANS cinerea (Butternut)

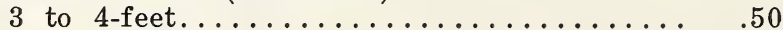

60.00

4 to 5 -feet. .

nigra (Black Walnut)

3 to 4 -feet..................... . 20

MAGNOLIA Lennei

4 to 5 -feet.

\section{Soulangeana}

4 to 5 -feet.

Stellata Halleana

$2 \frac{1}{2}$ to 3 -feet.

PLATANUS occidentalis (American Plane Tree)

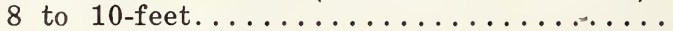

2 to $2 \frac{1}{2}$-inch.

POPULUS alba canescens (Green-leaved Poplar)

fastigiata (Lombardy Poplar)

8 to 10 -feet.

monolifera (Carolina Poplar)

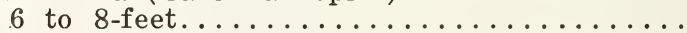

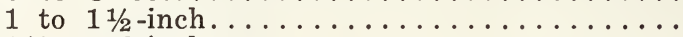

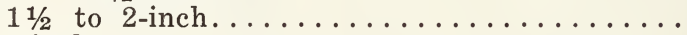

2 -inch

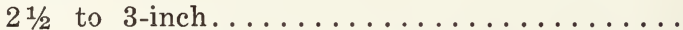

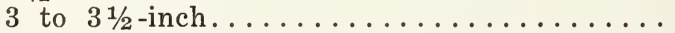

PRUNUS avium fl. pl. (Double Flowering Cherry)

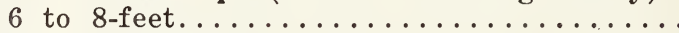

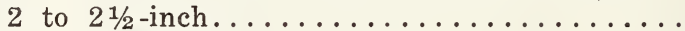

QUERCUS macrocarpa (Bur Oak)

3 to 4 -feet.

4 to 6 -feet.

palustris (Pin Oak)

4 to 6 -feet.

1.50

12.00

2.50

20.00

150.00

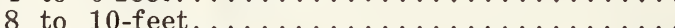
rubra (Red Oak)

4 to 5 -feet.

ROBINIA pseud-acacia (Black Locust)

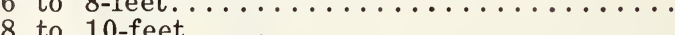

SALIX alba (White Willow)

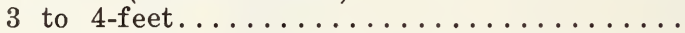

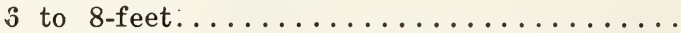

8 to 10 -feet.

Britzensis (Red Willow)

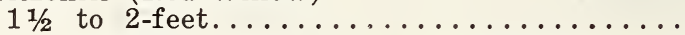

3.00

25.00

5 to 6 -feet.

pentandra (Laurel-Leaf Willow)

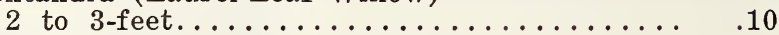

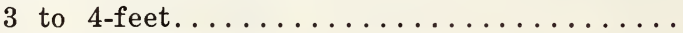

vitellina (Russian Golden Willow)

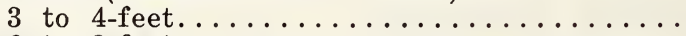

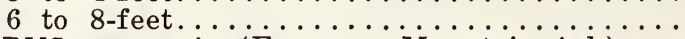

SORBUS aucuparia (European Mountain Ash)

6 to 8 -feet. 
TILIA Americana (Linden or Basswood)

Each

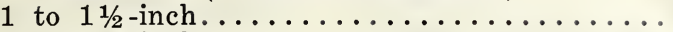

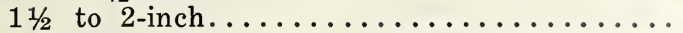

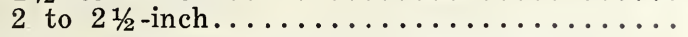

ULMUS Americana (American White Elm)

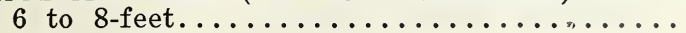

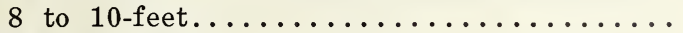

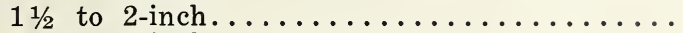

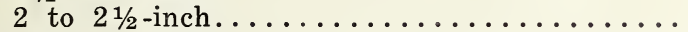

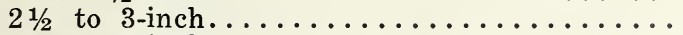

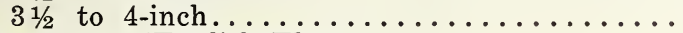

campestris (English Elm)

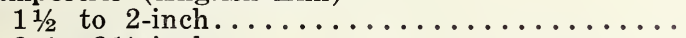

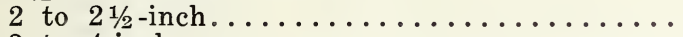

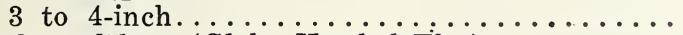

umbraculifera (Globe Headed Elm)

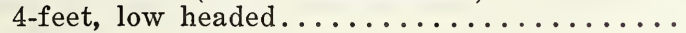

Wheatleyi

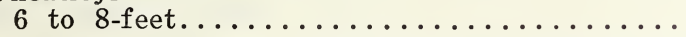

WE E P I N G TRE E S

MORUS tatarica pendula (Tea's Weeping Mulberry

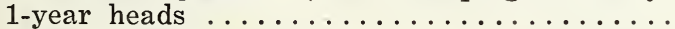

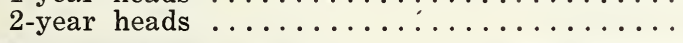

ULMUS montana var. pendula (Camperdown Elm) 5-year heads
8-year heads

APPLES

F R U I T S-(Bearing Size)

Duchess, Fameuse, Gano, Grimes' Golden, King, McIntosh, Northern Spy, N. W. Greening, Talman Sweet, Wealthy, Walbridge, Wolf River, Wisconsin Russet, Pewaukee, Red Astrachan, Yellow Transparent, Winesap, Peerless, Delicious, Prices' Sweet and Mam. Black Twig

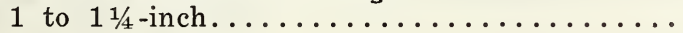

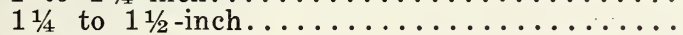

\section{CRABS}

Whitney, Florence, Hyslop, Red Siberian, Yellow

Siberian, Soulard and Transcendant $3 / 4$ to 1 -inch..................... .35

Hyslop

1 to $1 \frac{1 / 4}{\text {-inch }}$

\section{CHERRIES}

Early Richmond

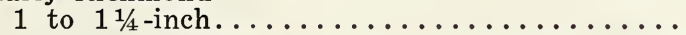

$11 / 4$ to $11 / 2$-inch.

\section{PEACHES}

Elberta, Champion, Early Crawford, Late Crawford, Carman and Salway

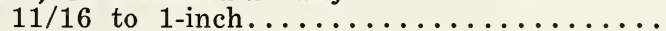

\section{PEARS}

Kieffer, Bartlett, Duchess, Flemish Beauty, Seckel and Dwarf Duchess

$3 / 4$ to 1 -inch...

PLUMS

$1 \frac{1}{4}$ to $1 \frac{1 / 2}{-i n c h}$

Lombard, German Prune, Bradshaw, Burbank, Shrop Damson, Abundance and DeSota

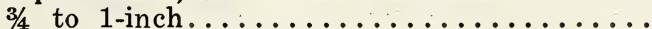

S MALL FRUITS

\section{BLACKBERRIES}

Snyder, Eldorado, Mersereau and Rathbun 


\section{GOOSEBERRIES}

Downing, Champion, Houghton, Josselyn and Industry

$$
\text { 2-year }
$$

Downing

\section{GRAPES}

\section{Concord}

2-year

3-year

Worden, Moore's Early, Niagara, Agawam and

Moore's Diamond

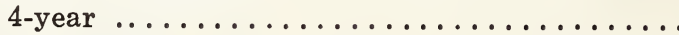

\section{RASPBERRIES}

Columbian, Cumberland and Golden Queen

2 -year ......................... .10 . $\quad .85$

STRAWBERRIES, (Everbearing)

Per 100 Per 1000

Americus, Progressive and Superb............ $\$ 2.50$

In variety ......................... 1.00

$\$ 15.00$

5.00

S H R U B S

ALTHEA in named varieties (Tree Form)

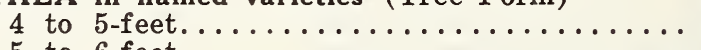

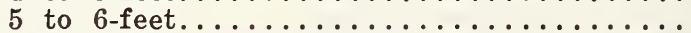

Each

Per 10

$\$ 0.50$

named varieties (Bush Form)

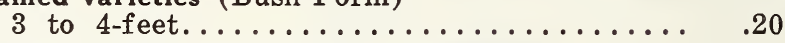

$\$ 3.00$

4.00

Per 100

$\$ 25.00$

35.00

$20 \quad 1.50$

12.00

AMELANCHIER Botryapium (Juneberry)

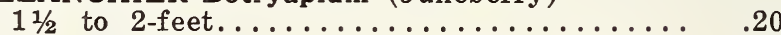

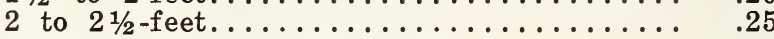

1.25

10.00

$25 \quad 1.50$

12.00

AMORPHA fruiticosa (False Indigo)

4 to 5 -feet.

10.00

ARALIA Pentaphylla

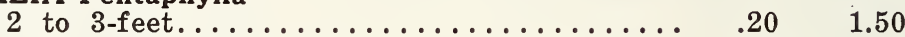

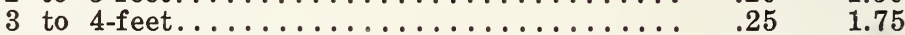

15.00

spinosa (Hercules' Club)

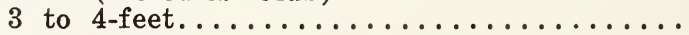

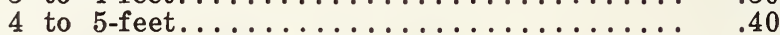

ARONIA arbutifolia (Red Chokeberry)

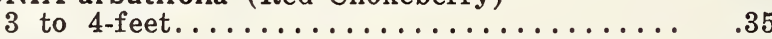

BERBERIS Japonica (Upright Thunberg's Barberry)

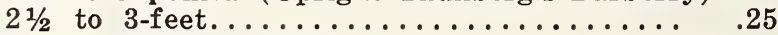

Thunbergii (Japanese Barberry)

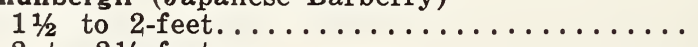

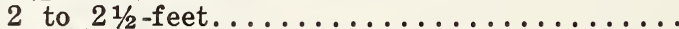


CEANOTHUS Americana (Red Root)

$11 / 2$ to 2 -feet.

Each

Per 10

Per 100

2 to $21 / 2$-feet.

CEPHALANTHUS occidentalis (Button Bush)

2 to 3 -feet.

10.00

\section{CERCIDIPHYLLUM Japonicum}

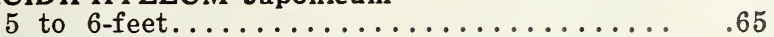

CLETHRA alnifolia (Sweet Pepper)

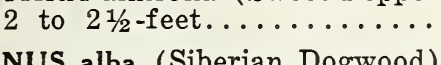

CORNUS alba (Siberian Dogwood)

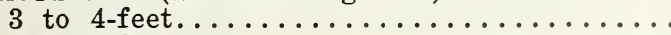

4 to 5 -feet.

5 to 6 -feet.

alternifolia (Blue Dogwood)

3 to 4 -feet..............

mascula (Cornelian Cherry)

paniculata (Gray Dogwood)

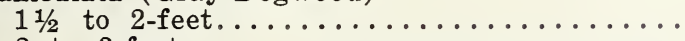

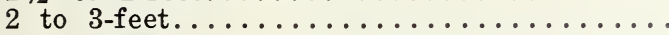

3 to 4 -feet.

sanguinea (Red Osier)

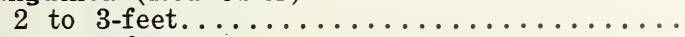

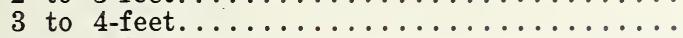

4 to 5 -feet.

sibirica (Red Dogwood)

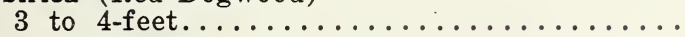

4 to 5 -feet.

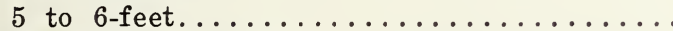

1.25

10.00

1.50

12.00

$2.00 \quad 15.00$

sericea (Silky Dogwood)

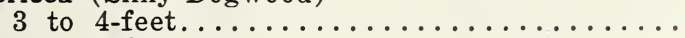

4 to 5 -feet.

stolonifera var. aurea (Yellow Dogwood)

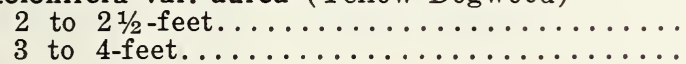

2.00

15.00

CRATAEGUS Crus Galli (Cockspur Thorn)

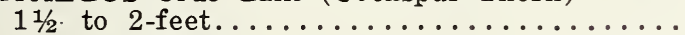

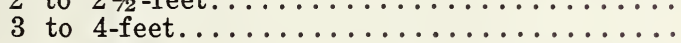

\section{coccinea}

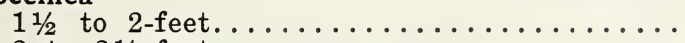

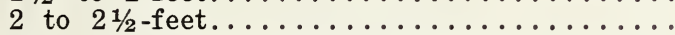

Paulii (Paul's Double Scarlet Thorn)
5 to 6 -feet.

6 to 8-feet.

CYDONIA Japonica (Japan Quince)

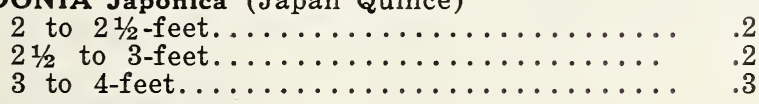

\section{DEUTZIA crenata}

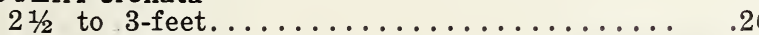

Pride of Rochester

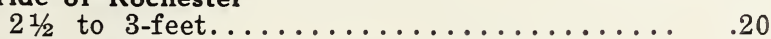

1.75

15.00

3 to 4 -feet.

$1.50 \quad 12.00$

ELEAGNUS angustifolia (Oleaster)

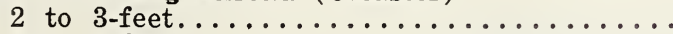

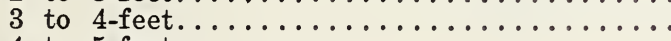


2 to 3-feet.

3 to 4-feet.

4.00

30.00

4 to 5 -feet.

\section{Europeus}

2 to 3 -feet

1.25

6 to 7-feet.

FORSYTHIA Fortuneii (Golden Bell)

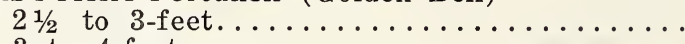

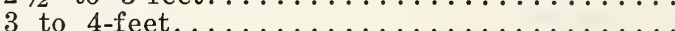

intermedia

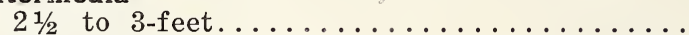

3 to 4 -feet.

.50

.15

4 to 5 -feet.

HIPPOPHAE Rhamnoides (Sea Buckthorn)

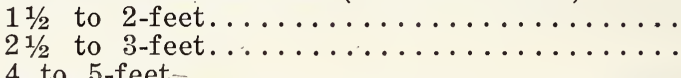

HYDRANGEA paniculata grandiflora (Tree Form)

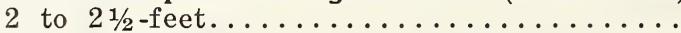

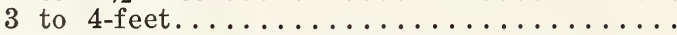

paniculata grandiflora (Bush Form) (Large Flowering Hydrangea)

$21 / 2$ to 3 -feet. . . . . . . . . . . . . . . . 18

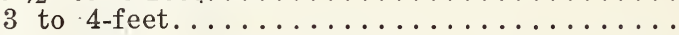

ILEX verticillata (Winterberry)

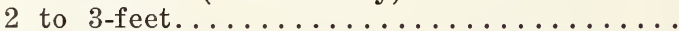

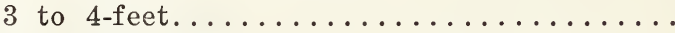

.20

.25

8.00

$1.25 \quad 10.00$

$1.50 \quad 12.00$

KERRIA JAPONICA

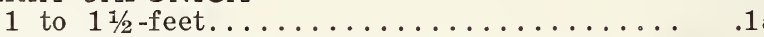

LIGUSTRUM Amurense (Amoor River Privet)

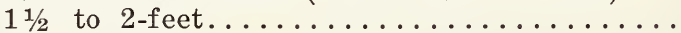

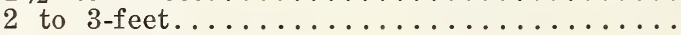

3 to 4 -feet.

$1.25 \quad 10.00$

ibota (Chinese Privet)

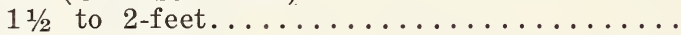

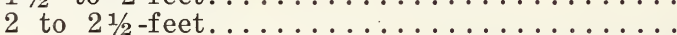

$1.50 \quad 12.00$

Regelianum (Regel's Privet)

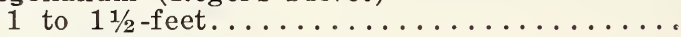

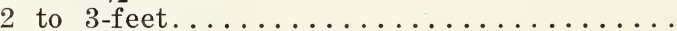

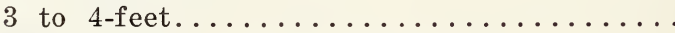

LONICERA alba (White Honeysuckle)

4 -feet $\ldots \ldots \ldots \ldots \ldots \ldots \ldots \ldots \ldots \ldots \ldots \ldots \ldots \ldots$. . . . . . . . . . . . regard this as the best variety of the Upright Honeysuckle grown, as it retains its good foliage throughout the summer, when other varieties are apt to be rusty. This is the best of all the Honeysuckles for hedges.

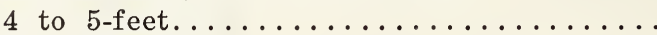

bella chrysantha

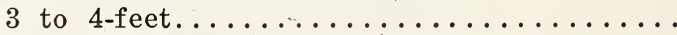

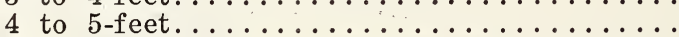

grandiflora (Pink Honeysuckle)

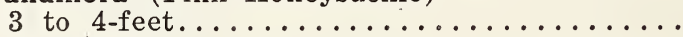

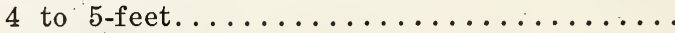

Morrowi

$$
3 \text { to } 4 \text {-feet. }
$$


MORUS alba (Russian Mulberry)

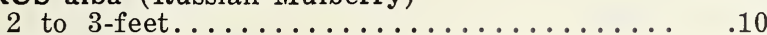

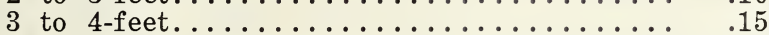

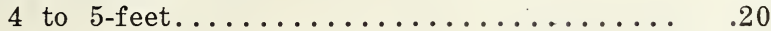

PHILADELPHUS coronarius (Mock Orange)

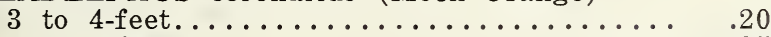

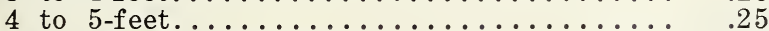

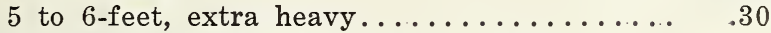

coronarius n'anus (Dwarf Mock Orange)

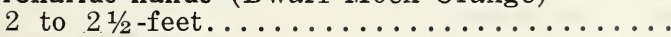

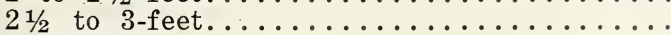

\section{Gordonianus}

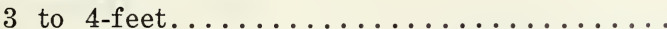

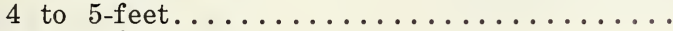

5 to 6 -feet.

grandiflorus

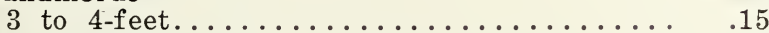

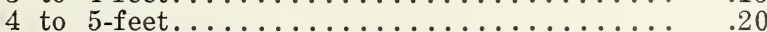

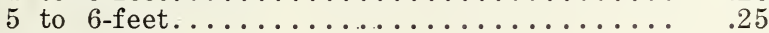

$1.00 \quad 8.00$

Lemoinei

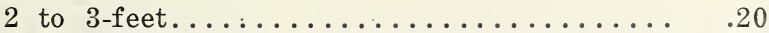

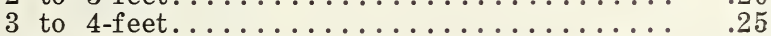

nivalis

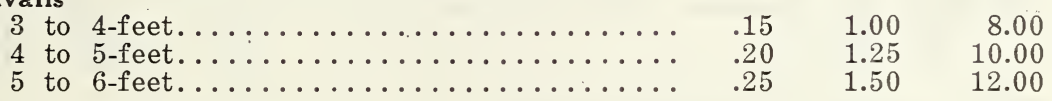

\section{POTENTILLA fruticosa}

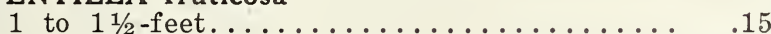

$1.25 \quad 10.00$

$1.50 \quad 12.00$

2 to 3 -feet.

PRUNUS Americana (Native Plum)

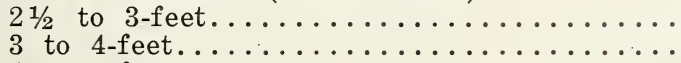

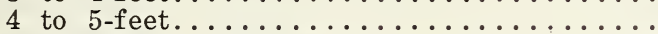

Besseyi (Rocky Mountain Cherry)

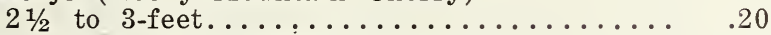

Othello Plum (Purple-leaved Plum)

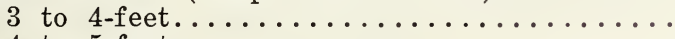

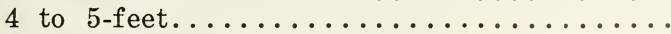

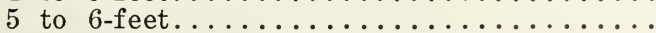

Pennsylvanica (Pin Cherry)

2 to 3 -feet..................... . 20

$1.75 \quad 15.00$

$2.50 \quad 20.00$

pissardi

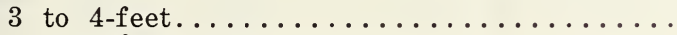

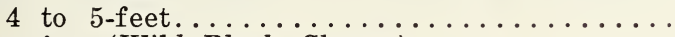

sorotina (Wild Black Cherry)

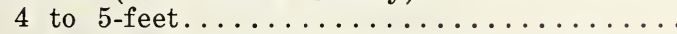

sinensis fl. pl. (Double Flowering Almond)

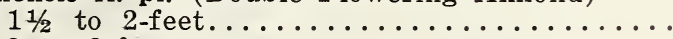

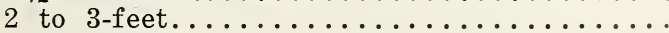

triloba (Flowering Plum)

$4.00 \quad 30.00$

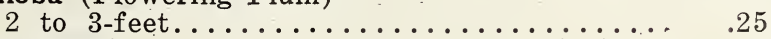

$2.00 \quad 17.50$

\section{Virginiana}

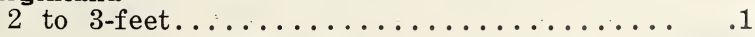

PTELEA trifoliata (Hop Tree) 
PYRUS angustifolia (Betchel's Double Flowering Each $\mathrm{Crab})$

3 to 4 -feet

coronaria (Wild Crab Apple)

$11 / 2$ to 2 -feet

2 to $21 / 2$-feet.

floribunda (Japanese Crab Apple)

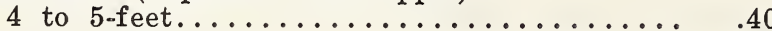

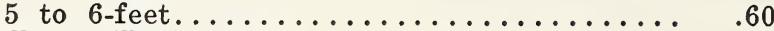

Halliana (Parkmanii)

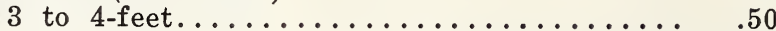

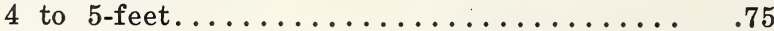

Niedzwetzkyana (Russian Crab Apple)

4 to 5 -feet.

Scheideckeri

4 to 5 -feet.

spectabilis (Chinese Flowering Apple)

3 to 4 -feet.

RHAMNUS cathartica

$21 / 2$ to 3 -feet. .

3 to 4 -feet.

4 to 5 -feet.

frangula

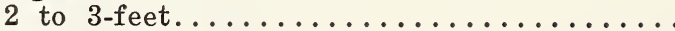

3 to 4 -feet

RHODOTYPOS kerrioides (White Kerria)

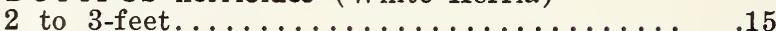

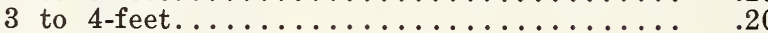

RHUS aromatica (Fragrant Sumach)

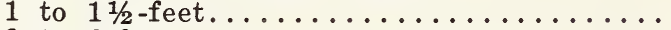

2 to 3 -feet.

copallina (Dwarf Shining Sumach)

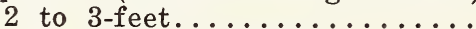

glabra (Smooth Sumach)

2 to 3 -feet................

typhina (Staghorn Sumach)

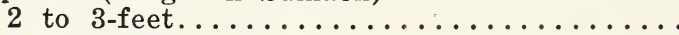

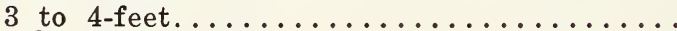

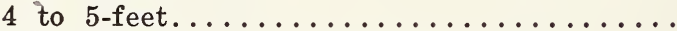

typhina laciniata (Cut-leaved Sumach)

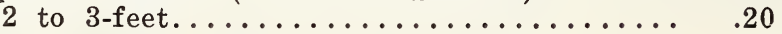

$11 / 2$ to 2 -feet.

aureum (Yellow Flowering Currant)

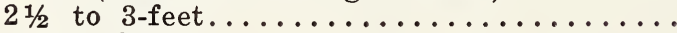

3 to 4 -feet.

\section{floridum}

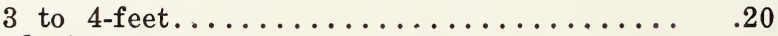

gordonianum

3 to 4 -feet.

4 to 5 -feet.

ROBINIA hispida (Moss Locust)

2 to 3 -feet

ROSA blanda

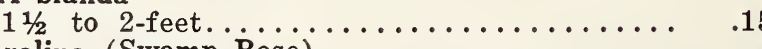

Carolina (Swamp Rose)

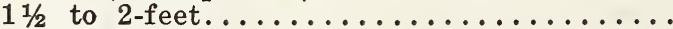

2 to 3 -feet.

humilis

$11 / 2$ to 2 -feet
.20

.25

.25

.20

.15

.20

.25

.20

.25

Per 10

Per 100

4.00

35.00

1.75

15.00

$2.50 \quad 20.00$

$3.50 \quad 30.00$

$5.00 \quad 40.00$

$4.00 \quad 35.00$

$5.00 \quad 40.00$

$40 \quad 3.00$

25.00

6.00

3.00

25.00

1.00

8.00

1.25

1.50

10.00

12.00

1.25

10.00

1.50

12.00

1.25

10.00

$1.50 \quad 12.00$

1.50

2.00

12.00

1.75

15.00

$1.25 \quad 10.00$

$\begin{array}{ll}1.00 & 8.00\end{array}$

1.50

12.00

1.75

15.00

$1.50 \quad 12.00$

.15

.20

1.50

12.00

$\begin{array}{ll}1.75 & 15.00\end{array}$

$1.25 \quad 10.00$

$1.50 \quad 12.00$

1.25

10.00

.15

1.25

10.00

.20

1.50

12.00

15.00 
lucida

$11 / 2$ to 2 -feet.

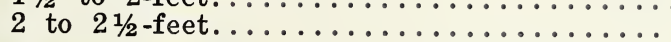

$.15 \quad 1.25$

$.20 \quad 1.50$

10.00

multiflora

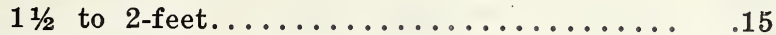

1.00

nitida

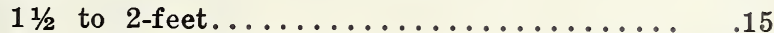

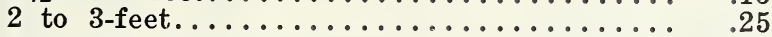

1.25

10.00

rubiginosa (Sweetbrier)

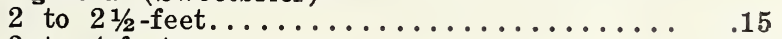

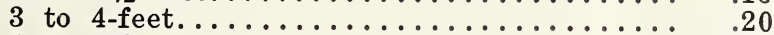

1.50

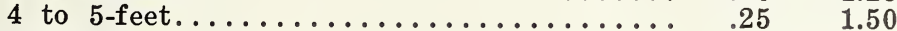

8.00

10.00

rubrifolia

3 to 4 -feet............................ 1.50

rugosa alba

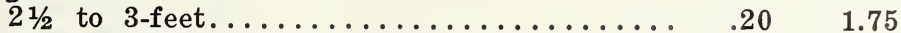

3 to 4 -feet.

rugosa (Red Japanese Rose)

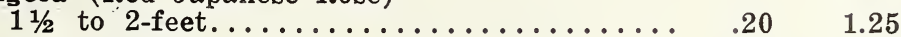

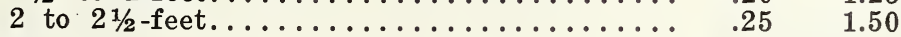

setigera

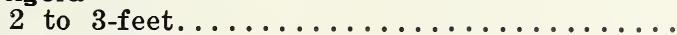

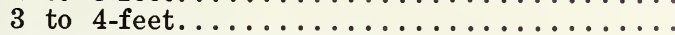

Wichuriana (Memorial Rose)

2 -year .

\section{L I M I I G and TRAIL I'N G ROSES}

American Beauty, red, 2-year........... .25

Crimson Rambler, red, 2-year............ $\quad .20$

Dorothy Perkins, pink, 2-year............ 20

Dorothy Perkins, white, 2-year............ $\quad .20$

Evergreen Gem, white, 2-year............ $\quad .20$

Gardenia, Bud bright yellow, opening cream, 2-year

Hiawatha, single red, 2-year.

Mme. Plantier, white, 2-year.............

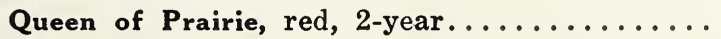

Tausendschon, pink, 2-year.

\section{HARDY PERPETUAL ROSES}

Anna de Diesbach, pink

Baron de Bonstetten, crimson

Conrad F. Meyer, pink

Frau Karl Druschki, pure white

Gen. Jacquiminot, brilliant crimson

Gruss an Teplitz, dark red

Magna Charta, pink and carmine

Marshall P. Wilder, red

Paul Neyron, deep rose

Prince Camille, de Rohan, velvety crimson

Ulrich Brunner, cherry red 


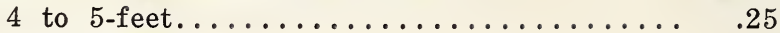

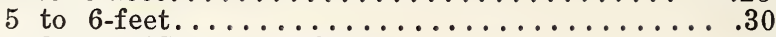

canadensis (Common Elder)

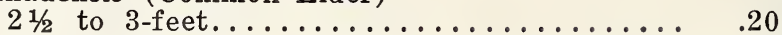

3 to 4 -feet.

canadensis laciniata (Common American Cutleaved Elder)

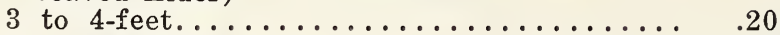

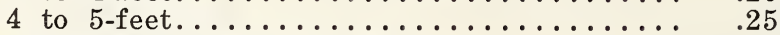

racemosus (Red-berried Elder)

$1.50 \quad 12.00$

3 to 4 -feet....................... 20

\section{SPIREA Anthony Waterer}

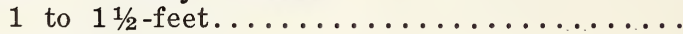

$11 / 2$ to 2 -feet

Bethlehemensis

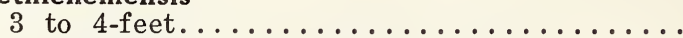

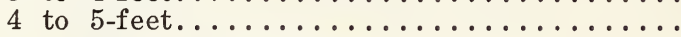

5 to 6 -feet, extra heavy.

\section{Billardii}

Bumalda

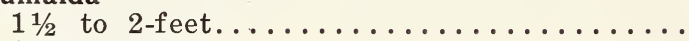

callosa froebeli

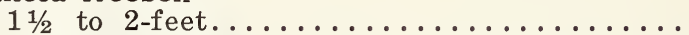

2 to $2 \frac{1}{2}$-feet

multiflora arguta

$1 \frac{1}{2}$ to 2 -feet.

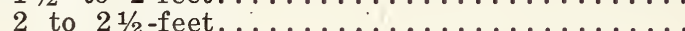
opulifolia

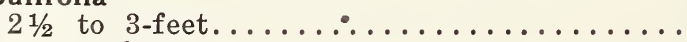

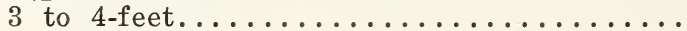

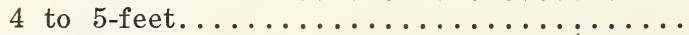

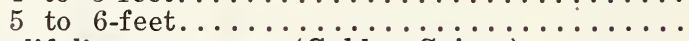

opulifolia var. aurea (Golden Spirea)

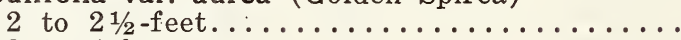

3 to 4 -feet.

paniculata

3 to 4 -feet. $\ldots \ldots \ldots \ldots \ldots \ldots \ldots \ldots$ sorbifolia

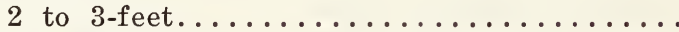
Thunbergii

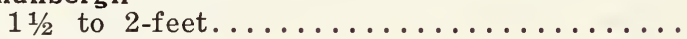

\section{Van Houtteii}

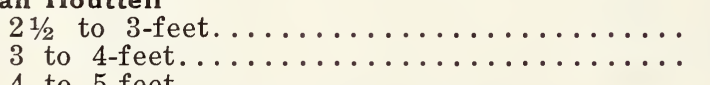

\section{SYMPHORICARPOS occidentalis (Wolfberry)}

3-feet racemosus (Snowberry)

2 to 3 -feet.

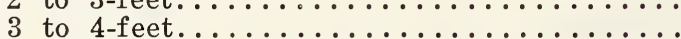
vulgaris (Indian Currant)

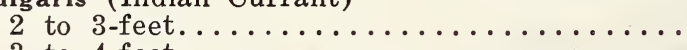

SYRINGA alba (White Lilac)

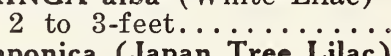

Japonica (Japan Tree Lilac)

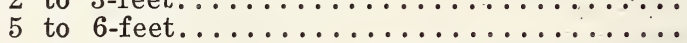

6 to 8 -feet. 
Persica alba (White Persian Lilac)

2 to 3 -feet........................... $25 \quad 2.00$

Rothomagensis (Persian Lilac)

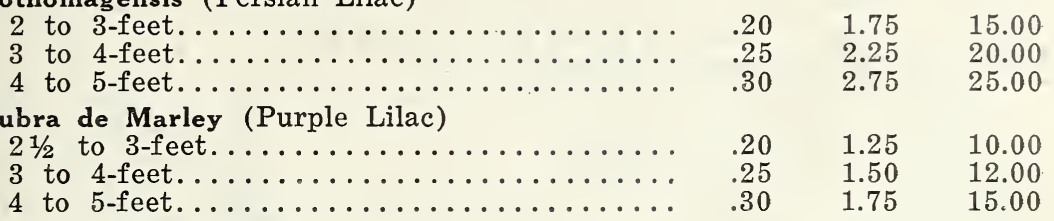

Marie Legraye, Souvenir Ludwig Spaeth, Charles X, and Mme. Lemoine

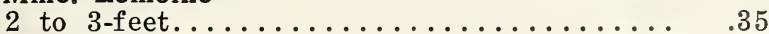

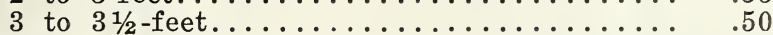

$3.00 \quad 25.00$

villosa

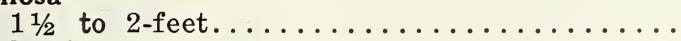

vulgaris

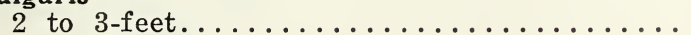

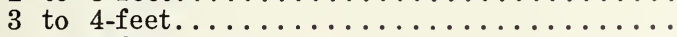

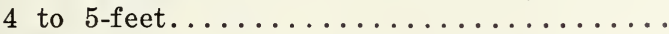

TAMARIX Amurense (Amoor River Tamarix)

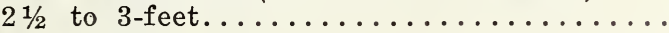

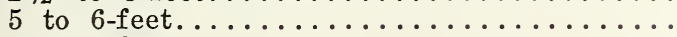

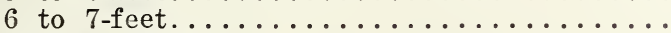

hispida aestivalis

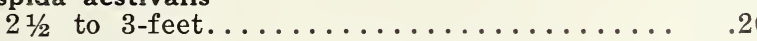

VIBURNUM dentatum (Arrow-wood)

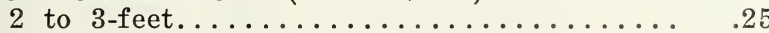

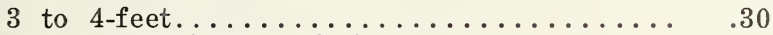

$3.50 \quad 30.00$

.25

$2.00 \quad 17.50$

.20

$1.25 \quad 10.00$

$1.50 \quad 12.00$

$.30 \quad 1.75 \quad 15.00$

.15

$1.00 \quad 8.00$

.25

.30

1.75

15.00

$2.50 \quad 20.00$

20

$1.50 \quad 12.00$

Lantana (English Wayfaring Tree)

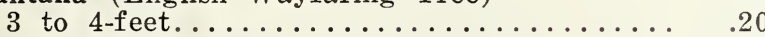

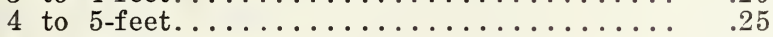

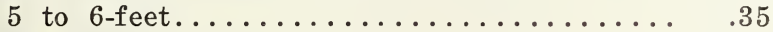

$2.00 \quad 18.00$

$2.50 \quad 20.00$

Lentago

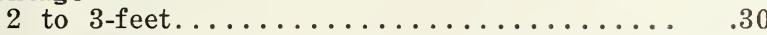

$1.50 \quad 12.00$

$2.00 \quad 18.00$

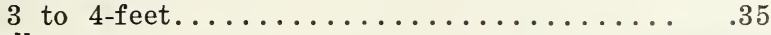

2.50

molle

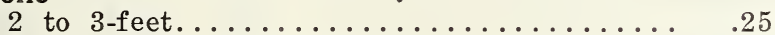

2.50

20.00

$3.00 \quad 25.00$

3 to 4 -feet.

$2.00 \quad 18.00$

.35

2.50

20.00

Opulus var. sterilis (Snowball)

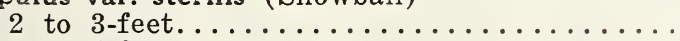

3 to 4 -feet.

.20

1.50

12.00

Opulus (H. B. Cranberry)

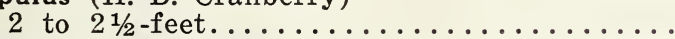

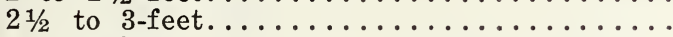

3 to 4 -feet.

4 to 5 -feet.

$1.75 \quad 15.00$

$\begin{array}{llr}.15 & 1.00 \quad 8.00\end{array}$

$.20 \quad 1.25 \quad 10.00$

$\begin{array}{lll}.25 & 1.50 & 12.00\end{array}$

plicatum

3 -feet

$2.50 \quad 20.00$

WEIGELA amabilis

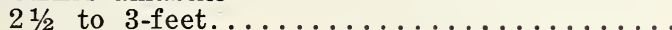

3 to 4 -feet.

Eva Rathke

2 to $2 \frac{1}{2}$-feet. .

3-feet

.30

.25

2.00

$.20 \quad 1.50$

12.00

$\begin{array}{lll}.25 & 1.75 & 15.00\end{array}$

$\begin{array}{lll}.25 & 2.00 & 17.50\end{array}$

$\begin{array}{lll}.35 & 2.50 \quad 20.00\end{array}$

rosea

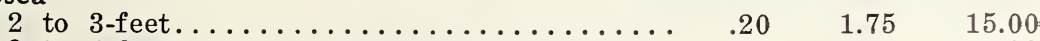

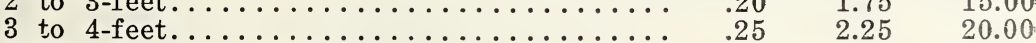

sessilif olia

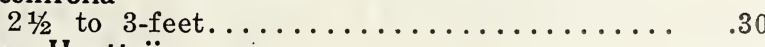

$2.50 \quad 20.00$

Van Houtteii

2 to 3 -feet. . 
XANTHOXYLUM Americanum (Prickly Ash)

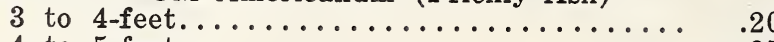

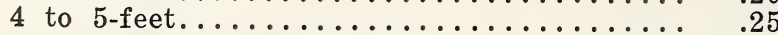

$1.50 \quad 12.00$

C L I M B I N G V I N E S AMPELOPSIS Engelmanni (Engelman's Ivy)

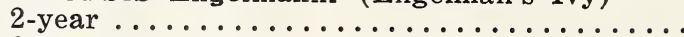

uinque folia (Virginia Creeper)

2-year

1.25

17.50

3-year ...

\section{Veitchi (Boston Ivy)}

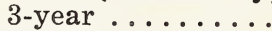

ARISTOLOCHIA Sipho (Dutchman's Pipe)

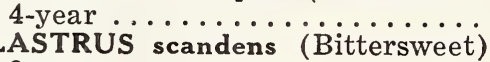

CLEMATIS paniculata

$2.00 \quad 15.00$

Henryi

$$
\text { 2-year }
$$

2-year

Jackmani

2-year

Mme. Edward Andre

2-year ........

vitalba

2 -year .

EUONYMUS vegetus

2-year. . . .....

\section{LONICERA Albertii}

$$
\text { 3-year }
$$

\section{Halleana}

punicea

3-year

LYCIUM Chinense (Matrimony Vine)

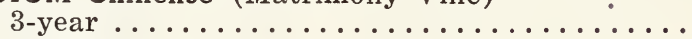

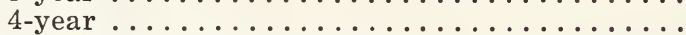

VITIS aestivalis (Wild Grape)

3-year.

WISTARIA Chinense (Chinese Blue Wistaria)

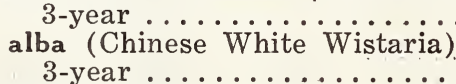

\section{LINNAEUS}

R H U B A R B

3-year

\section{S E E D L I N G S}

ULMUS Americana (American Elm)
$1 \frac{1 / 2}{2}$ to 2 -feet.

ASPARAGUS

2 -year.

ROBINIA pseud-acacia (Black Locust)

$21 / 2$ to 3 -feet....... LiNiNG OUT STOCK

PHILADELPHUS grandiflora

2-year cuttings

E V E R G E E N S

ABIES alba (White Spruce) Prices include B. \& B.

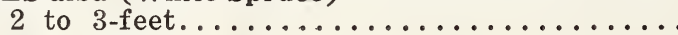

3 to 4 -feet. 
ABIES (Continued)

excelsa (Norway Spruce)

3 to 4 -feet..............

BUXUS Arborescens (Tree Box Bush Form)

12-inch

15-inch

Pyramidalis

$4 \frac{1}{2}$ to 5 -feet

5 to $51 / 2$-feet.

JUNIPERUS communis

$11 / 2$ to 2 -foot spread

2 to $2 \frac{1}{2}$-foot spread

Elegantissima Lee

Pfitzeriana

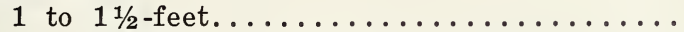

2 to 3 -feet.

Sabina

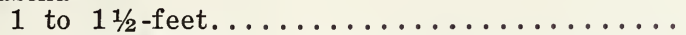

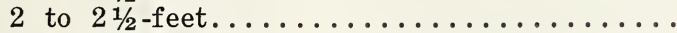

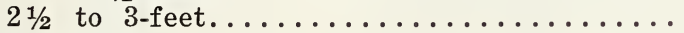

tamariscifolia

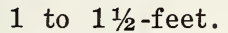

Virginiana (Red Cedar)

2 to 3 -feet.

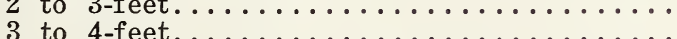

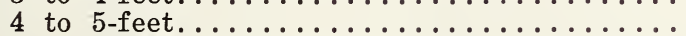

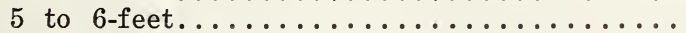

6 to 7 -feet.

7 to 8-feet. .

Virginiana var. glauca

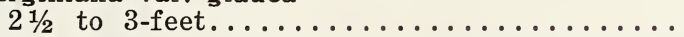

3 to 4 -feet.

PICEA pungens var. Kosterian'a (Koster's Blue Spruce)

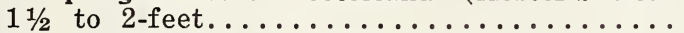

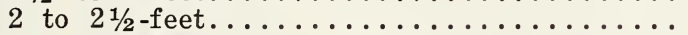

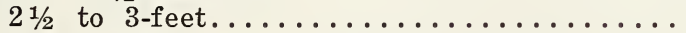

3 to 4 -feet.

4 to 5 -feet.

5 to 6 -feet.

6 to 7 -feet.

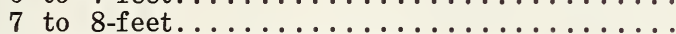

. . . . . . . . . . . .

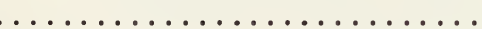

.........................

PINUS Austriaca (Austrian Pine)

$1 \frac{1 / 2}{2}$ to 2 -feet......

Mughus (Dwarf Pine)

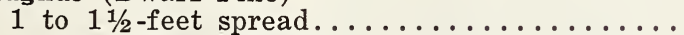

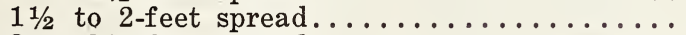

2 to $21 / 2$-feet spread.

resinosa

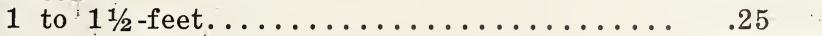

Strobus (White Pine)

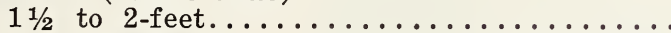

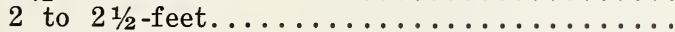

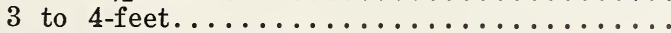

sylvestris (Scotch Pine)

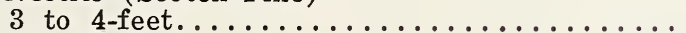

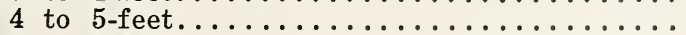

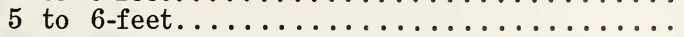

THUYA occidentalis (Arbor Vitae)

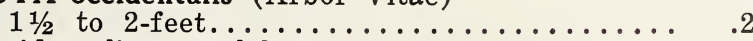

occidentalis var. globosa

1 to $11 / 2$-feet.

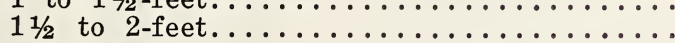

Each Per 10 Per 100

$\begin{array}{lll}1.00 & 7.50 \quad 50.00\end{array}$

$\begin{array}{lll}.50 & 4.00 \quad 35.00\end{array}$

$5.00 \quad 40.00$

7.00

$1.00 \quad 7.50$

65.00

$\begin{array}{lll}1.50 & 10.00 \quad 85.00\end{array}$

$.75 \quad 5.00$

$\begin{array}{rr}.75 & 5.00\end{array}$

$2.00 \quad 17.50$

$\begin{array}{ll}.75 & 5.00\end{array}$

$1.75 \quad 15.00$

$3.00 \quad 25.00$

$1.50 \quad 12.00$

$1.25 \quad 10.00$

$1.50 \quad 12.50$

$2.50 \quad 20.00$

$4.00 \quad 35.00$

$8.00 \quad 75.00$

$12.00 \quad 100.00$

$1.75 \quad 15.00$

$3.00 \quad 25.00$

$1.50 \quad 12.00$

$2.50 \quad 20.00$

$3.00 \quad 25.00$

$5.00 \quad 40.00$

$6.00 \quad 50.00$

$8.50 \quad 75.00$

$10.00 \quad 85.00$

$12.00 \quad 100.00$

$.50 \quad 4.00$

35.00

$.75 \quad 6.00$

50.00

$1.50 \quad 12.50$

$\begin{array}{ll}1.75 & 15.00\end{array}$

2.00

18.00

$.20 \quad 1.50$

12.00

$\begin{array}{lll}.50 & 3.50 & 25.00\end{array}$

$.75 \quad 5.00$

$.75 \quad 5.00$

35.00

$\begin{array}{rrr}1.00 & 7.50 & 50.00 \\ 1.25 & 10.00 & 75.00\end{array}$

$.20 \quad 1.75 \quad 15.00$

$\begin{array}{lll}.65 & 5.00 \quad 45.00\end{array}$

$\begin{array}{lll}1.00 & 7.50 & 65.00\end{array}$ 
cidentalis var. Pyramidalis

$11 / 2$-feet $\ldots \ldots \ldots \ldots \ldots \ldots$

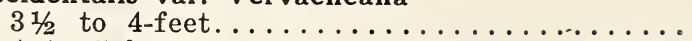

2.00

17.50

$2.50 \quad 20.00$

150.00

4 to 5 -feet.

H ARDY HER B A C O US PLA N T S

ACHILLEA Ptarmica (The Pearl) ........... .10

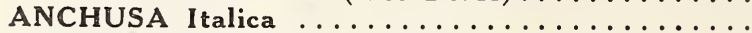

.15

ANTHEMIS Kelwayi (Golden Marguerite) ........

.10

tinctoria (Yellow)

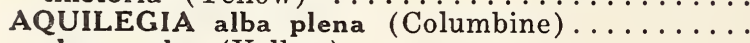

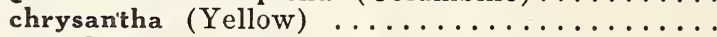
caerulea

vulgaris (Mixed Colors)

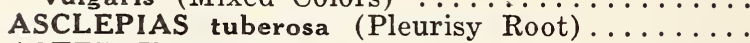

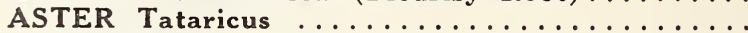

BOLTONIA asteroides (White)............

CAMPANULA Medium (Canterbury Bell).......

CENTAUREA atro purpurea (Corn Flower)....

CHRYSANTHEMUM Maximum (Shasta Daisy)...

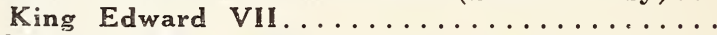

CONVALLARIA majalis (Lily of the Valley)....

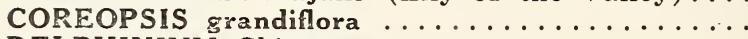

DELPHINIUM Chinense ..............

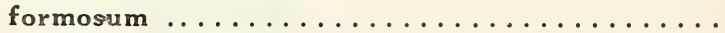

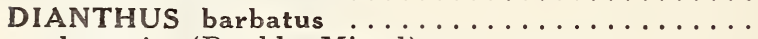

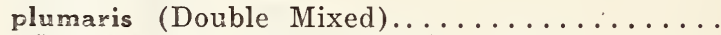

DIELYTRA spectabilis (Bleeding Heart)........

DIGITALIS Monstrosa (Fox Glove) ..........

FUNKIA subcordata

GAILLARDIA grandiflora . . . . . . . . . .

HEMEROCALLIS flava (Lemon Day Lily) ....... fulva (Orange Day Lily) ...............

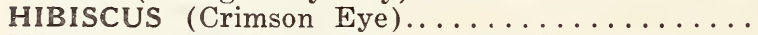

HIBISCUS (Meehan's Mallow Marvel) 2-year.....

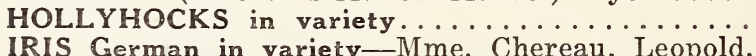

IRIS German in variety-Mme. Chereau, Leopold,
Chas. Dickens, Hamlet, Hector, Delmatica,

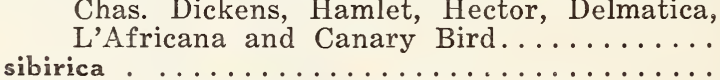

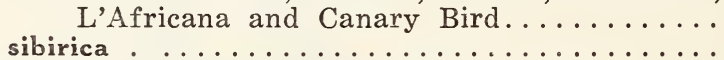

LYCHIIS chalcedonica (Jerusalem Cross).......

MONARDA didyma (Horse Mint) ............

PAPAVER Orientale

PEONIES in variety-Festiva, Louis Van Houtte, Modeste Guerin, Nobilissima, Lutea, Couronne d'Or, Reevesiana, Edulis superba, Purpurea superba, Duchess de Nemours, Duke

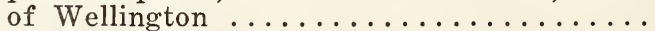

PLATYCODON grandiflora $\ldots \ldots \ldots \ldots \ldots \ldots$

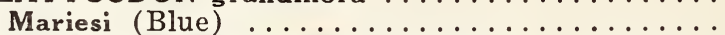

PHLOX in variety-Queen, Coquelicot, Bridesmaid, Champs Elysee, Eclaireur, R. P. Struther, Elizabeth Campbell, Baron van Dedem, Pink Beauty, Athis, Mme. Paul Dutrie........

PYRETHRUM roseum double.............

RUDBECKIA laciniata (Golden Glow).........

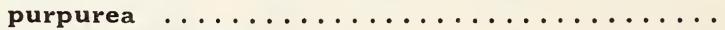

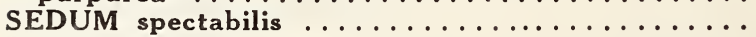

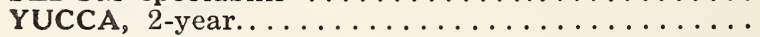

3 -year

.10

.15

.15

.15

.15

.15

.10

.15

.12

.15

.15

.15

.15

.10

.15

.15

.10

.10

.20

.15

.15

.15

.10

.15

.15

.20

.12

.80

1.00

.80

.80

1.00

1.00

1.00

1.00

1.00

.80

1.00

1.00

1.00

1.00

1.00

1.25

.80

1.00

1.00

.80

.80

1.75

1.25

1.25

1.00

.80

1.00

1.00

1.50

1.00

175.00

.15

.15

.10

.15

.15

1.25

1.25

.80

1.00

1.25

6.00

8.00

6.00

6.00

8.00

8.00

8.00

8.00

8.00

6.00

8.00

8.00

8.00

8.00

10.00

6.00

8.00

8.00

6.00

6.00

15.00

10.00

10.00

8.00

6.00

8.00

8.00

12.00

8.00

.50

.10

3.50

25.00

.15

.80

6.00

1.00

10.00

10.00

6.00

8.00

10.00

.15

1.00

.15

.10

.10

.15

.15

.20

1.25

.80

.80

1.25

1.00

1.25

8.00

G R A S S E S

EULALIA Gracillima ...............

Japonica 


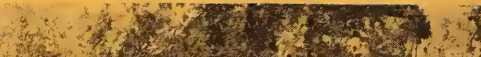

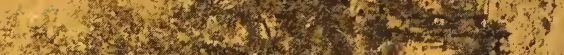

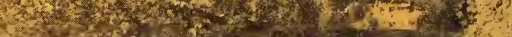

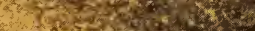

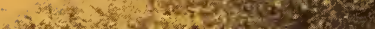

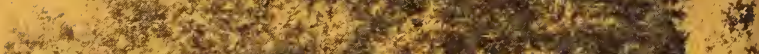
2.

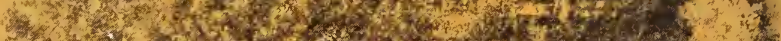

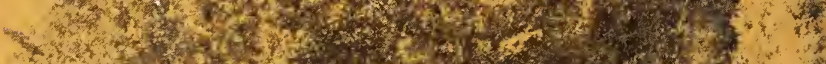

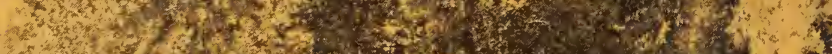

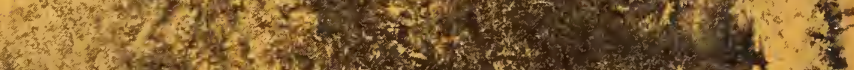

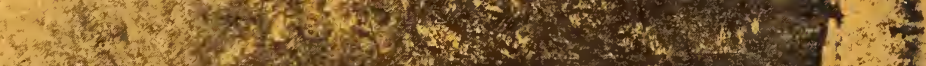

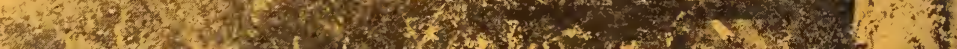

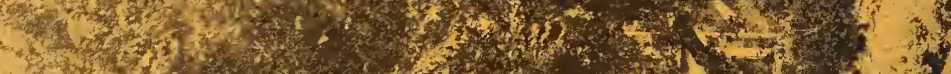

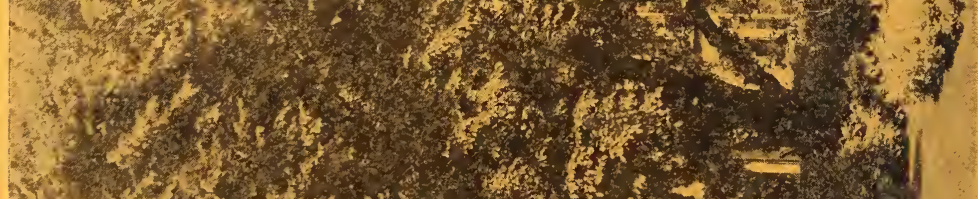

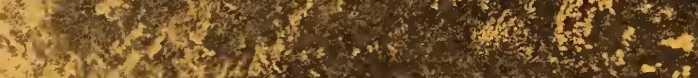

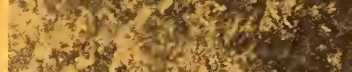

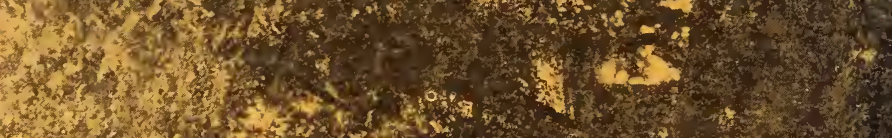

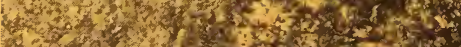

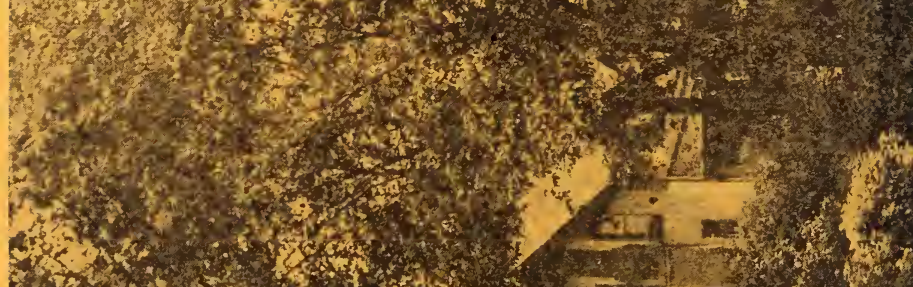

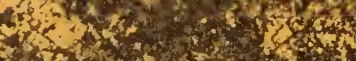

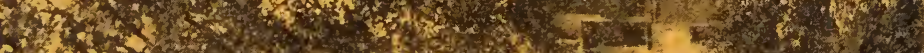

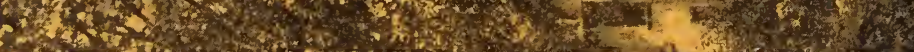

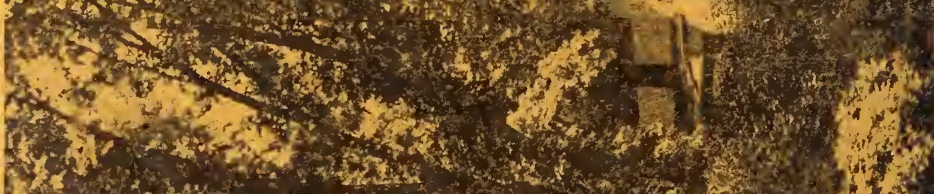

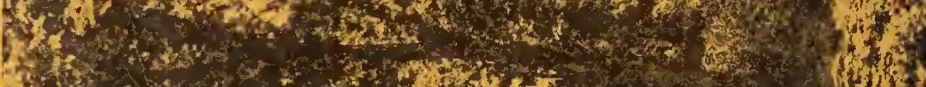
M.

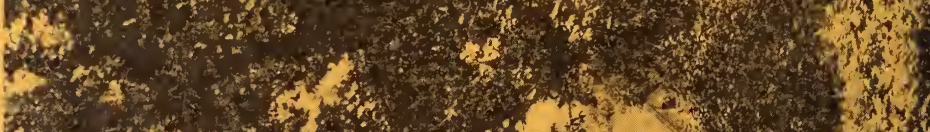

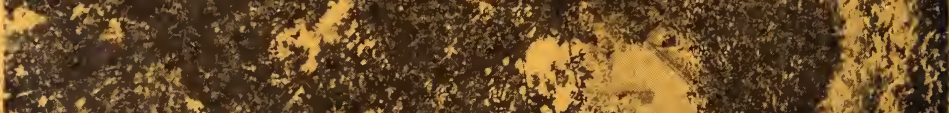

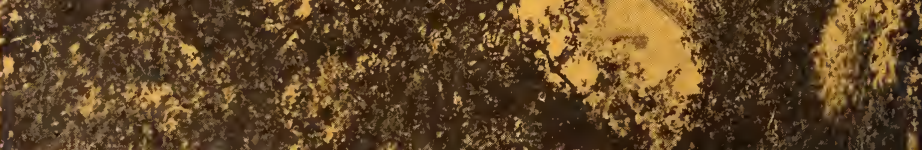

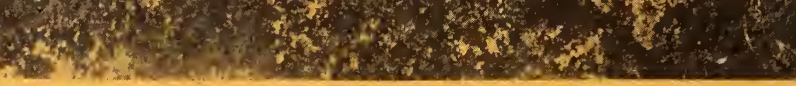

